Article

\title{
A Molecular Dynamics Study of Crosslinked Phthalonitrile Polymers: The Effect of Crosslink Density on Thermomechanical and Dielectric Properties
}

\author{
Janel Chua ${ }^{1}$ and Qingsong Tu ${ }^{2, *}$ \\ 1 DSO National Laboratories, Singapore 118225, Singapore; csonglin@dso.org.sg \\ 2 Department of Civil and Environmental Engineering, University of California, Berkeley, CA 94720, USA \\ * Correspondence: howietu@berkeley.edu
}

Received: 6 December 2017; Accepted: 8 January 2018; Published: 11 January 2018

\begin{abstract}
In this work, molecular dynamics (MD) and molecular mechanics (MM) simulations are used to study well-equilibrated models of 4,4'-bis(3,4-dicyanophenoxy)biphenyl (BPh)-1,3bis(3-aminophenoxy)benzene ( $\mathrm{m}$-APB) phthalonitrile (PN) system with a range of crosslink densities. A cross-linking technique is introduced to build a series of systems with different crosslink densities; several key properties of this material, including thermal expansion, mechanical properties and dielectric properties are studied and compared with experimental results. It is found that the coefficient of linear thermal expansion predicted by the model is in good agreement with experimental results and indicative of the good thermal stability of the PN polymeric system. The simulation also shows that this polymer has excellent mechanical property, whose strength increases with increasing crosslink density. Lastly and most importantly, the calculated dielectric constant-which shows that this polymer is an excellent insulating material-indicates that there is an inverse relation between cross-linking density and dielectric constant. The trend gave rise to an empirical quadratic function which can be used to predict the limits of attainable dielectric constant for highly crosslinked polymer systems. The current computational work provides strong evidence that this polymer is a promising material for aerospace applications and offers guidance for experimental studies of the effect of cross-linking density on the thermal, mechanical and dielectric properties of the material.
\end{abstract}

Keywords: phthalonitrile polymer; cross-linking density; molecular dynamics; thermal expansion; dielectric constant

\section{Introduction}

In the past 50 years, a considerable amount of research has been conducted on high temperature polymers for aerospace applications. For a long time, most high temperature polymer composite materials were based on polymeric systems which are functional up to $\sim 400{ }^{\circ} \mathrm{C}$. Applications that required an operating temperature of $>400{ }^{\circ} \mathrm{C}$ had to rely on denser high temperature materials such as ceramic systems or metallic alloys. A breakthrough came, however, when Keller from Naval Research Laboratory [1] started developing thermosetting phthalonitriles for potential aerospace, marine and electronic packaging applications at temperatures $>400{ }^{\circ} \mathrm{C}$. Unlike most of its thermoset predecessors which cure by a process that creates a lot of voids due to release of volatile by-products, phthalonitrile polymer systems are formed via the addition cure mechanism where phthalonitrile based monomers react with aromatic amines such as 1,3-bis(3-aminophenoxy)benzene (m-APB) or bis[4(4-aminophenoxy)phenyl]sulfone (p-BAPS) to produce a highly-crosslinked system. This curing method ensures that little to no volatiles are evolved during polymerization leading to highly crosslinked, void-free polymer networks that have good mechanical properties: with almost the 
same specific compressive strength as concrete and only $6 \%$ the density of concrete when in its foamed form [2,3]. Besides its exceptional mechanical properties, this material also shows outstanding thermo-oxidative stability at elevated temperatures and superior fire resistance [1]. In addition, there has been an increased interest in studying the electrical properties of phthalonitrile with fillers (such as graphene flakes or silver nano-particles) to explore its potential application in microscale composite electrical devices where high mechanical strength, low density and good electrical properties are of interest [4]. In order to investigate the aforementioned properties of the PN polymer system, directed trial and error approaches could be employed to optimize the experimental processing conditions. Such methods are however potentially time-consuming and expensive. In order to save on natural and human resources, researchers have turned to computer simulations as a means to understanding these polymer systems. On one hand, they can reduce trial and error experimentation; on the other hand, they provide insights at the molecular and micro length scales which are often difficult to obtain from experiments.

One of the polymer systems that were used as a pioneering test case in molecular modelling of polymers was the epoxy system [5]. Over the last 20 years, MD simulations based on bead-spring models and Monte-Carlo simulations based on bond-fluctuation model have been used to study the molecular behavior of polymeric materials [6,7]. Most of these studies were however primarily mathematical in nature and did not take into account the topological information on the network. As a result of this, useful information related to mechanical as well as thermodynamic properties was not accounted for. Soon after, several authors studied the formation of crosslinked epoxy with topological information using different approaches of simulated cross-linking. Doherty et al. [8] created polymethacrylate (PMA) networks using lattice-based simulations using the polymerization molecular dynamics scheme. Yarovsky et al. [5] discussed a methodology to cross-link low molecular weight water soluble phosphate-modified epoxy resin (CYMEL 1158). However, all the cross-linking reactions were carried out simultaneously (static cross-linking) in a three-step procedure in their study. Cross-linking of epoxy resins using molecular dynamics was performed by $\mathrm{Xu}$ et al. [9] and their model was used to study diffusion of water in epoxy's crosslinked networks. The authors used an iterative Molecular Dynamics (MD)/Molecular Minimization (MM) procedure to crosslink an epoxy resin where the newly formed topology was subjected to 1000 MD steps of relaxation, with one crosslink established per iteration. Other computational studies involving epoxies [10-12] have been conducted.

Although the aforementioned studies have made significant progress in the molecular modelling of crosslinked epoxies, similar models have not been developed for additional cured polymeric systems that contain multiple cross-linking steps and dielectric properties of crosslinked polymeric systems have also not been widely studied at the molecular length scale. Thus, in the spirit of the above discussion, a molecular dynamics study on a crosslinked phthalonitrile polymeric system was conducted. In the paper, we examined PN polymer, a thermally stable resin synthesized by Keller from USA Naval Research Laboratory [1] which was later successfully made into a carbonized foam by Liying Zhang et al. from Nanyang Technological University, Singapore [2]. An efficient method of creating a molecular model of crosslinked phthalonitrile system containing 10,000 atoms is described. Three models were built with different percentages of crosslink and the model predicts that the thermal expansion coefficient and elastic properties are dependent on crosslink density. Due to the potential electromagnetic interference (EMI) properties of carbonized phthalonitrile polymer [2], dielectric properties were also obtained for pre-carbonized crosslinked resin to serve as a reference point for future carbonization studies.

The paper is organized as follows. In Section 2, the molecular modelling is presented with the main focus on cross-linking procedure and systems with different cross-linking densities are modelled and discussed. In Section 3, material properties including thermodynamic, elastic and dielectric are presented as a function of crosslink density. Due to specific interest in the superior thermal and mechanical properties of phthalonitrile polymeric systems, the first two parts look at MD simulated thermal and mechanical properties. In the third part, the dielectric properties of pre-carbonized 
phthalonitrile polymers are studied in order to observe the change in dielectric constant as a function of carbonization in future work. A method for obtaining the dielectric constant of polymers using MD was established which will be applied to future work on carbonized phthalonitrile polymeric systems.

\section{Material and Molecular Modelling}

\subsection{Simulation Details}

The PN resin system modelled in this paper consists of the 4,4'-bis(3,4-dicyanophenoxy)biphenyl $(\mathrm{BPh})$ monomer and the cross-linking agent 1,3-bis(3-aminophenoxy)benzene (m-APB). The molecular structure of BPh and activated m-APB are shown in Figure 1.

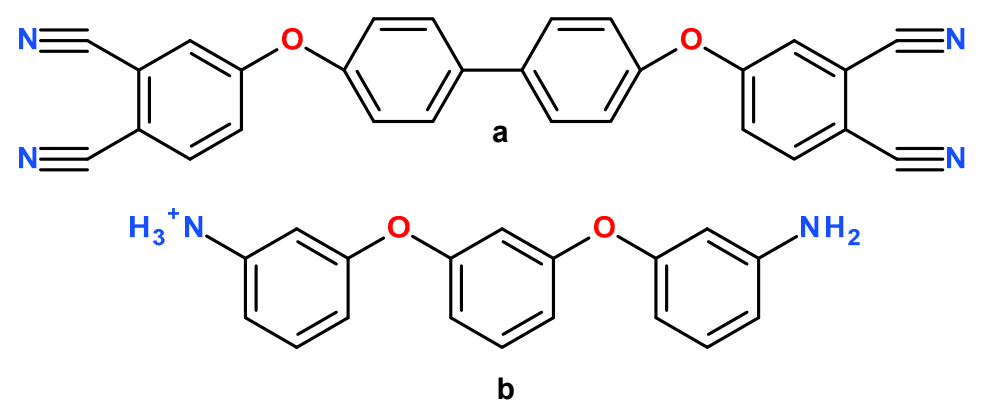

Figure 1. Molecular structure of (a) BPh monomer and (b) activated m-APB cross-linking agent.

The initial uncrosslinked molecular model structure was established using a procedure similar to that used by Varshney et al. [12]. In accordance with experimental measurements conducted by Keller et al. [13] a molar ratio of 28:1 (BPh:m-APB) with a density of $1.2 \mathrm{~g} / \mathrm{cm}^{3}$ is selected in this study. Image of the initial uncrosslinked structure along with a 3D molecular structure of the cross-linking agent and monomer are shown in Figure 2 below. The 3D structure is shown in the left and a cross section of the middle is shown on the right, curing agents are initially homogeneously distributed without any cross-linking. A homogeneous distribution was selected as it most closely mimics macroscale experimental conditions where we may assume that the curing agents are somewhat evenly distributed in the polymeric system. Should the distribution of curing agents be inhomogeneous, the effect in real life experiments and in modelled systems would be lower levels of cross-linking at saturation. This is due to the uneven cross-linking at the initial stages of cross-linking which results in steric hindrance at later stages of cross-linking. In our current study, we consider the case of a homogeneously distributed initial configuration in order to maximize the cross-linking levels at saturation.

In view of the charged $\mathrm{NH}_{3}{ }^{+}$present on the cross-linking agent, a substitute monomer was used for calculations due to the problems that will occur when periodic boundaries are used for charged systems. The substitute monomer does not change the cross-linking procedure and has no effect on the final configuration of the polymer as the monomer on which $\mathrm{NH}_{3}{ }^{+}$resides is a curing agent and will be removed at the end of the curing reaction; refer to Figure 3a for the curing reaction. The details of the substitute cross-linking agent are provided in supporting information.

The above uncrosslinked model contains a total number of 11,040 atoms, with only $8 \mathrm{~m}-\mathrm{APB}$ molecules and $224 \mathrm{BPh}$ molecules, to satisfy the ratio of $28: 1$. This model is further equilibrated through Molecular Dynamics (MD) method in order to construct the crosslinked structure in the next step. The equilibration is performed using LAMMPS molecular dynamics software [14]. Consistent Valence Force Field (CVFF) is used in all simulations $[15,16]$. The non-bonded van der Waals interactions are modelled as the 12-6 Lennard Jones (LJ) potential. The initial structure was formed in a $50 \times 50 \times 50 \AA$ simulation box with 3D periodic conditions and equilibrated using two MM minimization, one canonical ensemble (NVT) and one isothermal-isobaric (NPT) simulation at $300 \mathrm{~K}$ and atmospheric 
pressure for $100 \mathrm{ps}$. This is in order to minimize internal forces resulting from the construction of bonds, bond angles and bond dihedrals [12]. After obtaining the equilibrated structure for the uncrosslinked model, the following cross-linking procedure is introduced to construct the final model, which starts with assumptions needed in the procedure, followed by a flow chart of the algorithm and saturation.

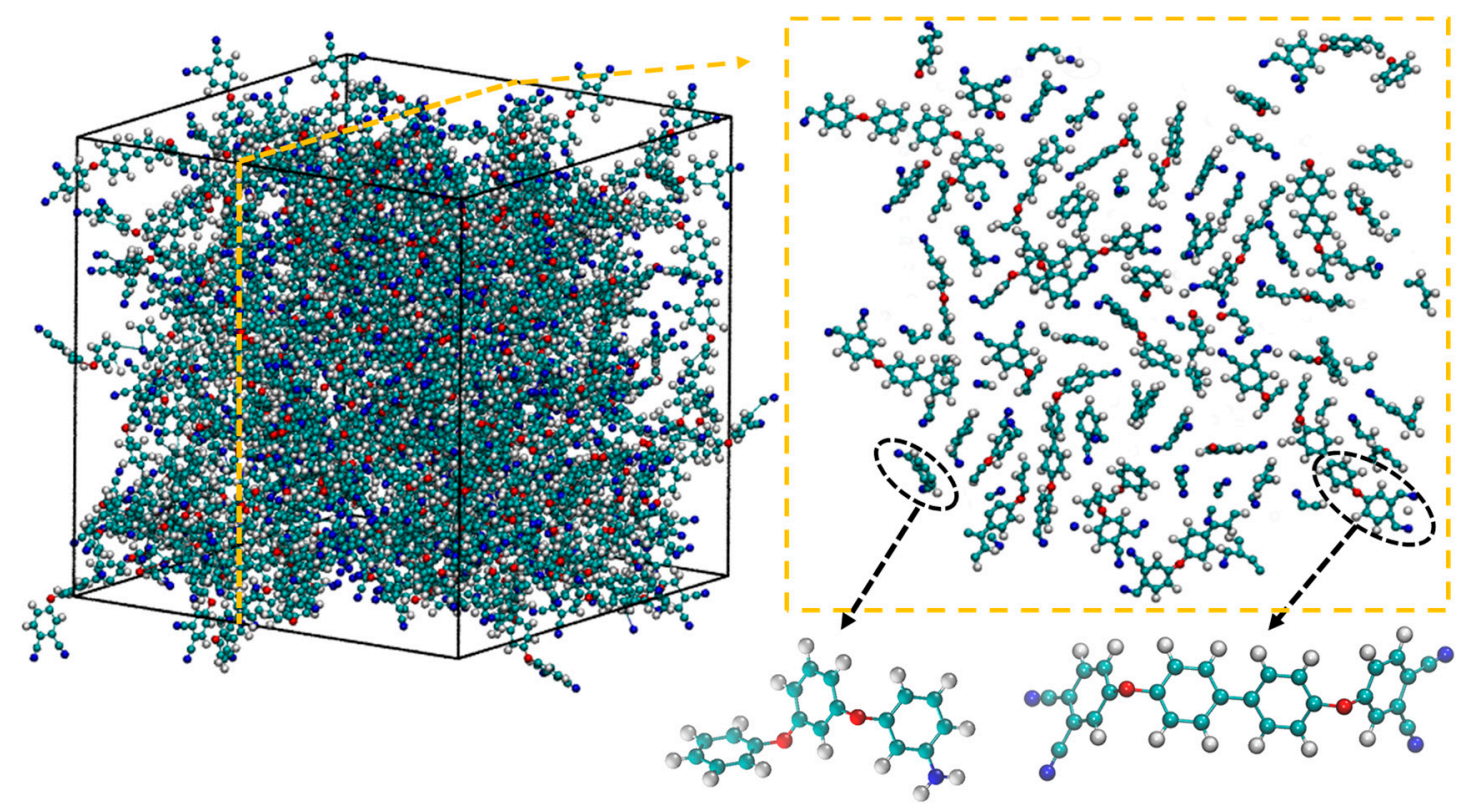

Figure 2. Initial uncrosslinked model of BPh-substitute m-APB mixture with ratio 28:1 ("red" represents Oxygen, "white" is Hydrogen, "blue" is Nitrogen and "green" is Carbon); 3D structure is depicted on the left and cross-section of the middle is depicted on the right.

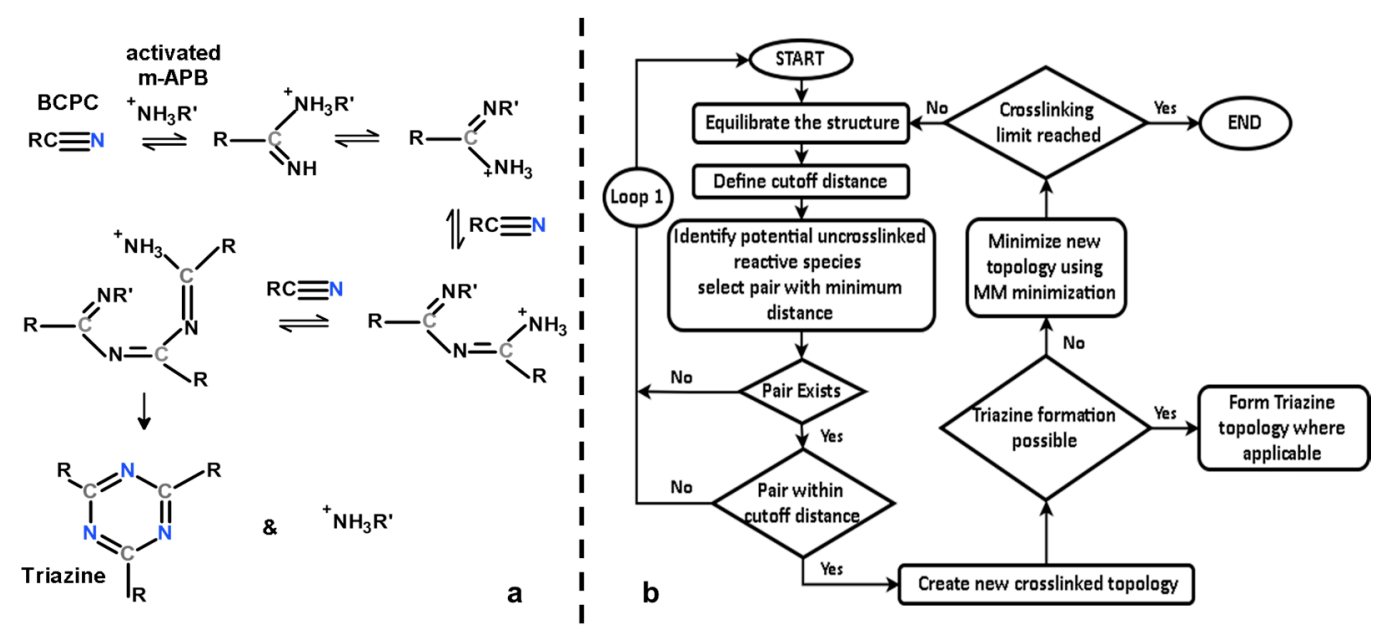

Figure 3. (a) Cross-linking reactions leading up to triazine formation; (b) cross-linking algorithm.

\subsection{Cross-Linking Procedure}

The cross-linking reaction of the phthalonitrile system involves the potential formation of a polytriazine, polyphthalocyanine or polyisoindolenine [17]. In our current study, we only considered the reaction pathway involving the formation of polytriazine; refer to Figure 3 for a schematic of the steps leading up to a triazine formation.

The equilibrated structure was statically crosslinked based on the distance between the $\mathrm{N}$ atom of the $\mathrm{NH}_{3}{ }^{+}$group $\left(\mathrm{NH}_{2}\right.$ in the substituted m-APB) in the activated m-APB cross-linking agent or 
molecule subsequently (after reaction with BPh monomer) and the $\mathrm{C}$ atom of the $\mathrm{C} \equiv \mathrm{N}$ group in the $\mathrm{BPh}$ monomer. While the actual cross-linking reaction is quite complex, the fundamental mechanisms of the reaction that were modelled in this work are depicted in Figure 3a, in accordance with the cross-linking mechanism established by Burchill [17] where monomers would crosslink via addition polymerization in the presence of a catalyst and undergo trimerization via a condensation reaction forming a triazine. In order to simplify the cross-linking process without losing any important structural information pertaining to the crosslinked structure at each step of the process, the following assumptions were made:

(1) All activated $\mathrm{m}$-APB were assumed to have the same reactivity.

(2) All $\mathrm{NH}_{3}{ }^{+}$reactive groups $\left(\mathrm{NH}_{2}\right.$ in substitute $\mathrm{m}-\mathrm{APB}$ ) were assumed to have the same reactivity no matter where their position is on the crosslinked monomer.

(3) A reaction will take place as long as at least one pair of reactive species are within the cutoff distance.

(4) Triazines will form instantaneously at any point in the cross-linking reaction once conditions for its formation are met.

A flowchart describing the cross-linking algorithm is shown in Figure $3 \mathrm{~b}$ and is discussed below.

Step 1: System is equilibrated using NPT simulations at $600 \mathrm{~K}$ (to imitate the curing temperature) for 40 ps. It is important to ascertain that the simulation time in between successive cross-linking reactions is long enough for unreacted species to move around. This simulation time was chosen based on the calculated root-mean-square displacement (RMSD). The RMSD of reaction carbon and nitrogen atoms were found to be $\sim 4.6$ and $4.2 \AA$ in 40 ps respectively [12], thus ensuring that the equilibrium time was long enough for various molecules to mix.

Step 2: Initial cutoff distances are defined for the cross-linking algorithm. If and only if reactive species exist within the cutoff distance, the cross-linking reaction will proceed. Atom pairs with minimum distances were selected to create new crosslinked topology due to the relationship between interatomic potential energy and interatomic distance. Reactive atoms (that are at distances $\geq r_{m}$, where $r_{m}$ is the distance at which the potential reaches its minimum) experience greater attraction with smaller distances which would in turn increase their chances of forming bonds. Thus, the cross-linking methodology was based on having reactive pairs with the minimum distance forming bonds.

Step 3a: If cross-linking does occur, the topological information is updated by introducing new bonds into the system. The system will be scanned for potential structures that are able to form triazines and if those structures exist, the triazine structure will be formed and the activated cross-linking agent will be released back into the system. Thereafter, the topology is relaxed via MM minimization.

Step 3b: If cross-linking does not occur, the system is further equilibrated according to Step 1.

Step 4: After minimization, if cross-linking limit is not reached, the algorithm jumps to Step 1 where the equilibration of the new structure is performed, otherwise, simulations are stopped.

In order to study the properties of the polymer system with respect to cross-linking density, we first define the cross-linking limit as the total number of crosslinks formed in the polymer system when the cutoff distance is set to the space diagonal of simulation box. The crosslink \% for other cutoff distances where cutoff distance <space diagonal of simulation box was then defined to be the ratio of the total number of crosslinks that were formed to the cross-linking limit (maximum number of crosslinks possible for that system). Saturation is defined to occur when Loop 1 loops for three consecutive times, at which point, no more cross-linking can take place without extreme perturbation of the system and cross-linking is considered complete.

Once a crosslink is created, charge distribution around the reacted atoms will be altered. In order to account for that, charges were evaluated on a model molecule built with Insight II [15] which has a molecular topology similar to the newly formed cross-link. The original charge distribution was then replaced with the appropriate charges in order to keep the system neutral. The details of the charge redistribution are provided in supporting information. 
In order to achieve a relaxed network, the crosslinked system is further equilibrated with one MM minimization, heating at $600 \mathrm{~K}$ with NVT equilibration, compression at $600 \mathrm{~K}$ and $9000 \mathrm{~atm}$ external pressure using NPT equilibration followed by quenching with NPT dynamics to $300 \mathrm{~K}$ and $1 \mathrm{~atm}$ external pressure. Representative cross-link densities that represent the expected range for a stoichiometric monomer/cross-linking agent ratio were then chosen for subsequent modeling steps.

\section{Results and Discussion}

Figure 4 shows the cross sections of the final equilibrated structures (for clarity, only in-plane molecules are depicted). Monomers that are already crosslinked are grouped together. Comparing the cross section of $25 \%, 50 \%$ and $100 \%$ crosslinked structure, we found that: (a) The number of circled clusters decreases as the cross-linking density increases, which means monomers are increasingly crosslinked together, from totally uncrosslinked (depicted in Figure 2) to 100\% crosslinked (depicted in Figure 4c) which is the formation of one big molecule from all the linked monomers; (b) the density of the system increases as cross-linking level increases because monomers become closer to each other. The volume of the system shrinks gradually during the process of network formation, as observed in experiments. For highly dense system ( $100 \%$ cross-linking), a volume shrinkage of about $7 \%$ (relative to its volume at $0 \%$ cross-linking) is observed in our simulations. This is comparable with $12.8 \%$ reported by Keller [18] for phthalonitrile resin heated at $500{ }^{\circ} \mathrm{C}$ for $24 \mathrm{~h}$.
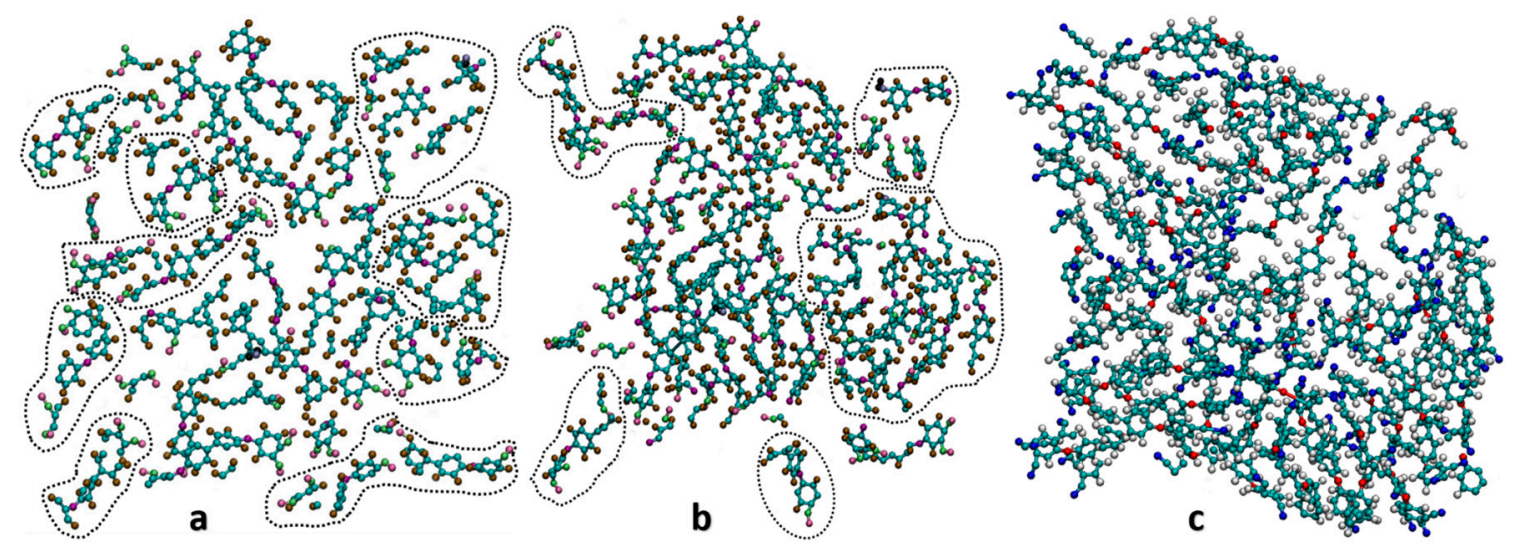

Figure 4. Cross sections of crosslinked phthalonitrile system with (a) $25 \%$, (b) $50 \%$ and (c) $100 \%$ cross-linking. In $(\mathbf{a}, \mathbf{b})$, different clusters of polymers that are un-crosslinked are circled by dash lines.

\subsection{Thermal Properties}

Thermal expansion coefficients were used to study the thermal property of this material. Volume change $\mathrm{dV}$ with respect to the initial volume $V_{0}$ (at $300 \mathrm{~K}$ ) for the $25 \%, 50 \%$ and $100 \%$ crosslinked systems in the temperature range 300-900 K were determined and plotted in the Figure 5 below. Each crosslinked system was equilibrated at $1 \mathrm{~atm}$ for 30 ps before an NPT (constant temperature and constant pressure) simulation was conducted where the polymer was slowly heated from $300 \mathrm{~K}$ to $900 \mathrm{~K}$. From the plot, it is clear that the $50 \%$ crosslinked polymer system exhibited a significantly larger volume expansion than the $100 \%$ crosslinked system. This is due to the reduced number of covalent bonds in systems with lower cross-linking densities, which will allow more monomers to move around freely.

Linear regression lines were fitted on the volume expansion curves in Figure 5 to determine the coefficient of volumetric thermal expansion (CVTE) in both the rubbery and glassy regimes. In the glassy regime, the data were fitted for the $300 \mathrm{~K}-450 \mathrm{~K}$ temperature range and for the rubbery regime, the data were fitted for $750 \mathrm{~K}-850 \mathrm{~K}$. Given that the $T_{\mathrm{g}}$ of phthalonitrile polymer systems is around $670 \mathrm{~K}-700 \mathrm{~K}$, the coefficient of linear thermal expansion (CLTE) was then calculated using a method similar to that used by Ananyo et al. [10]. 


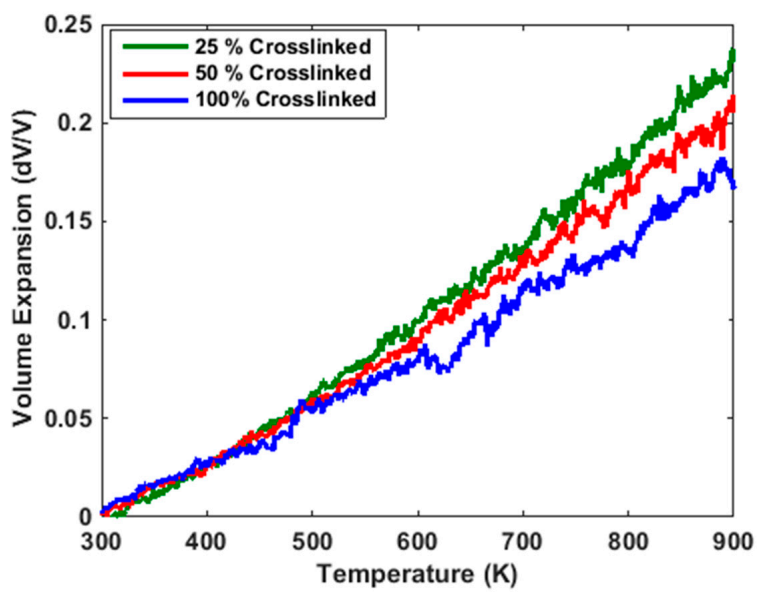

Figure 5. Volume expansion with respect to temperature for 25\%,50\% and 100\% crosslinked structures.

The CLTE values of the crosslinked systems are shown in Table 1 and generally indicate an increase in the coefficient of expansion with decreasing crosslink density which is expected due to increased mobility of molecule chains at lower crosslink densities. The results were found to be consistent with those reported by Itecma [19] which reported an experimental CLTE value of $9.0 \times 10^{-5} \mathrm{~K}^{-1}$ when the $\mathrm{PN}$ resin was postcured to $180^{\circ} \mathrm{C}$ and a value of $3.5 \times 10^{-5} \mathrm{~K}^{-1}$ when the resin was postcured to $375^{\circ} \mathrm{C}$.

Table 1. Coefficients of linear thermal expansion under different cross-linking densities, in unit $10^{-5} \mathrm{~K}^{-1}$.

\begin{tabular}{cccc}
\hline CLTE & $\mathbf{2 5 \%}$ & $\mathbf{5 0 \%}$ & $\mathbf{1 0 0} \%$ \\
\hline Above $T_{\mathrm{g}}$ & 15.13 & 13.70 & 12.86 \\
Below $T_{\mathrm{g}}$ & 9.99 & 8.77 & 6.99 \\
\hline
\end{tabular}

\subsection{Mechanical Properties}

Simulated compression tests were performed on $25 \%$ crosslinked, $50 \%$ crosslinked and $100 \%$ crosslinked systems to determine their elastic properties. In these simulations, uniaxial compressive strain and uniaxial tensile strain with a strain rate of $10^{12} / \mathrm{s}$ were imposed on the MD models with periodic boundary conditions in the NPT ensemble. NPT simulations were run at $300 \mathrm{~K}$ for $10 \mathrm{ps}$. Strain increments were applied at every timestep such that the desired cumulative strain was reached by the end of the simulation. The elastic responses of polymers are generally dependent on applied strain rate $[20,21]$ (with the young's modulus increasing by up to 4 times in the case of PMMA when strain rate was varied from $\sim 10^{-3} / \mathrm{s}$ to $\sim 10^{3} / \mathrm{s}$ ) and it is to be noted that for the current MD simulations, the strain rate used is very much higher than experimental strain rates which are typically on the order of $10^{-4} / \mathrm{s}$. As a result, the strain rate hardening which results from a much higher strain rate explains the discrepancy between experimental specific tensile strengths of $\sim 36 \mathrm{MPa} \cdot \mathrm{cm}^{3} / \mathrm{g}$ [19] compared to the computed specific compressive strength shown in the graph below of $\sim 1000 \mathrm{MPa} \cdot \mathrm{cm}^{3} / \mathrm{g}$.

By comparing the results of the system at different cross-linking percentages in Figure 6 below, it is apparent that the yield stress of the $100 \%$ crosslinked system is the highest followed by $50 \%$ crosslinked and $25 \%$ crosslinked. The percentage strain at which the maximum yield stress occurred is noted to decrease with increasing crosslink percent. This is in agreement with the typical behavior of polymeric systems, with more highly crosslinked systems having a greater load bearing ability than systems that are not as highly crosslinked [22]. The higher strain percentage at which systems with lower levels of crosslink yield is also in accordance with polymeric behavior where the greater the molecular weight of the molecule chains, the stiffer the polymer will be, resulting in brittle behavior with yield occurring at low strain percentages [23]. This trend is reflected in the Young's modulus by calculating the slope of the linear part of curves in Figure 6b, with values of $143.4 \mathrm{GPa}$, 174.0 GPa and 
$201 \mathrm{GPa}$, for $25 \%, 50 \%$ and $100 \%$ crosslinked PN respectively. The increasing tendency of Young's modulus is indicative of higher stiffness for higher crosslink densities.

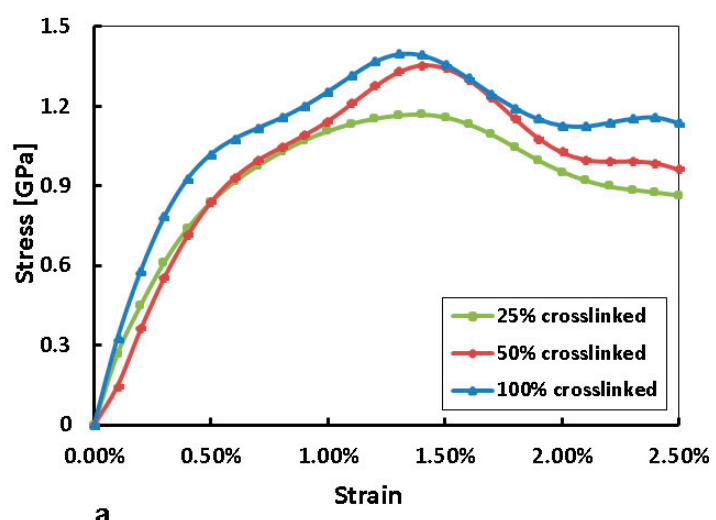

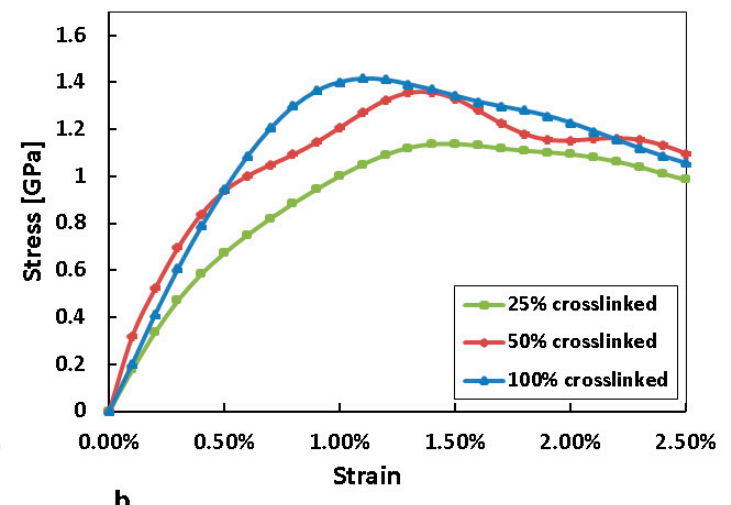

b

Figure 6. Graph of stress versus strain at different crosslink \% (a) tensile strength; (b) compressive strength.

\subsection{Dielectric Properties}

We proceed with the calculation of the static dielectric constant using the different crosslinked systems. Fix E-field command in LAMMPS was used in the molecular dynamics simulation for an empirical approximation to how charged particles react in the presence of an external E-field. For the polymeric system under uniaxial displacement, the dielectric constant can be defined in Equation (1).

$$
k_{11}=k_{0}\left(1+\chi_{11}\right)
$$

$k_{11}$ is the dielectric constant along axial direction, $k_{0}=8.854 \times 10^{-12} \mathrm{~F} / \mathrm{m}$ is the vacuum permittivity, $\chi_{11}$ is the susceptibility of the medium, which is a function of axial polarization density $P_{1}$ and the E-field applied to the material:

$$
\chi_{11}=\frac{1}{k_{0}} \frac{\partial P_{1}}{\partial E_{1}}
$$

The axial polarization $P_{1}$ is determined from $P_{1}=P_{1}^{e}+P_{1}^{d}$, where $P_{1}^{e}$ is the polarization due to the relative displacement of electrons and core and $P_{1}^{d}$ denotes the polarization due to the relative displacement between atoms. Following the molecular dynamics simulation done by Zhang [24] the effect of polarization between the nucleus and electron cloud is neglected in this study, which results in elimination of the clamped ion term i.e., $P_{1}^{e}=0$. The axial polarization vector thus can be written as:

$$
P_{1}=P_{1}^{d}=\sum_{i=1}^{N} \frac{x_{1}^{i} q_{i}}{\bar{V}}
$$

where $q_{i}$ and $x_{1}^{i}$ are, respectively, the electric charge and the coordinate along the axial direction of atom $i, N$ is the number of atoms and $\bar{V}$ is the volume of the polymer mass.

To calculate dielectric constant, different E-field values are applied across one axis of the PN model, the structure is relaxed and the coordinates of all its atoms in the final relaxed state is recorded. The polarization densities under different $E$ values are obtained according to Equation (3). A $P_{1}-E_{1}$ curve is obtained and the slope equals to the electrical susceptibility $\chi_{11}$ defined in Equation (2). A detailed derivation and steps to get $\chi_{11}$ are shown in supporting information.

The $P_{1}-E_{1}$ curves of different crosslinked systems are plotted in Figure 7. 


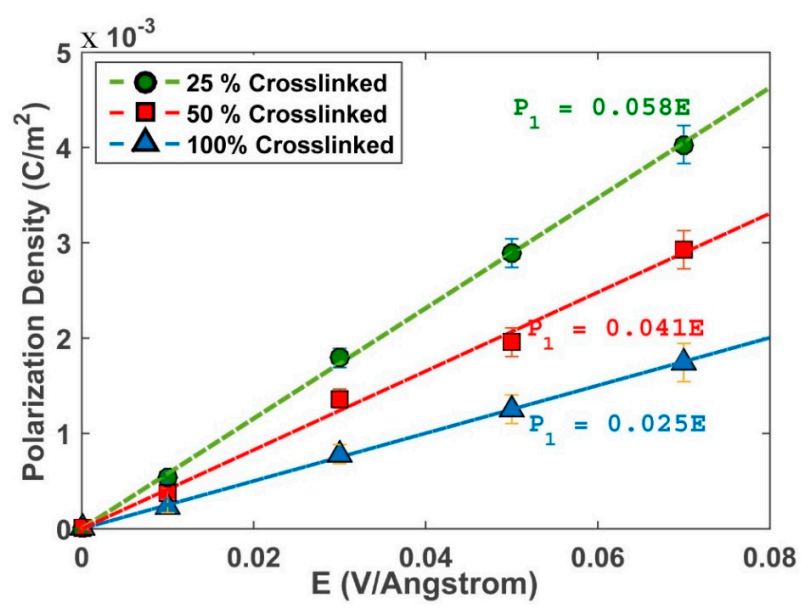

Figure 7. Graph of normalized polarization density versus electric field for different crosslinked systems.

As expected, a linear relation between electric field and polarization density is found, whose slope is proportional to the susceptibility of the system $\chi_{11}$; this value decreases as the cross-linking level increases, because the material becomes rigid and less polarizable upon the imposition of an electric field. Converting $\chi_{11}$ to dielectric constant through Equation (1), we get the final dielectric constants of different systems, as shown in Figure 8.

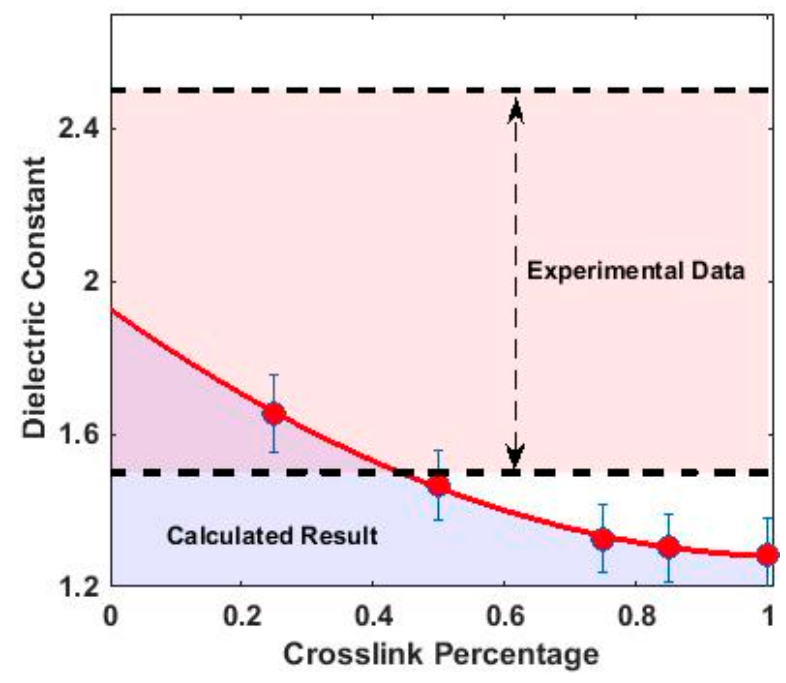

Figure 8. Graph of dielectric constant versus \% crosslink.

Dielectric constants calculated above are indicative of an insulating material. The value decreases as the level of crosslink density increases and an empirical quadratic function $\mathrm{D}=0.57 \rho^{2}-1.22 \rho+1.92$ can be used to fit the relation between dielectric constant (D) and cross-linking density $(\rho)$. A comparison was conducted between calculated values and experimental data of similar PN systems $[4,25,26]$ and intersections between them were found for lower crosslink densities.

As shown in Figure 8, the calculated dielectric constant is relatively low compared to the experimental range. This is probably due to the symmetry of triazine structures modelled in this work as well as the decrease in mobility of structures with higher crosslink densities. Under laboratory conditions, it is difficult to control the trimerization of crosslinked monomers. Not every crosslinked monomer will form a triazine structure and the polymeric system will likely have triazine and linear chains of crosslinked monomers existing together. The polarizability of the system will increase with the increase of asymmetrical linear chains due to a net polarization which explains the increasing difference 
between experimental results and modelled results as cross-linking percentage increases. Additionally, a $100 \%$ crosslinked polymer structure hinders orientational polarization due to its decreased molecular mobility. The polymer mixes that were used to obtain the experimental dielectric constant were unlikely to be $100 \%$ crosslinked systems, contributing to increased orientational polarizability of molecule chains, leading to a higher measured dielectric constant. As such, the calculated dielectric constant for a $100 \%$ crosslinked polymer system provides a useful upper limit reference for the above modelled phthalonitrile polymer system.

\section{Conclusions}

The present article provides a detailed and clear discussion on the molecular modelling study of a crosslinked BPh-m-APB phthalonitrile polymeric system modelled to crosslink densities that are difficult to attain experimentally ( $100 \%$ crosslinked). This material shows excellent mechanical property with high strength and low dielectric constant.

We also found that the level of crosslink density is a very important factor affecting the material's properties. As the cross-linking density increases, the material shows better resistance to mechanical deformation, and becomes a better insulator with a lower dielectric constant. We also studied the limiting case of $100 \%$ cross-linking via computational methods, which shows the best mechanical and electric properties; in this aspect, we are able to extend the understanding of this material, overcoming experimental limitations of synthesizing a 100\% crosslinked polymer.

Although the procedure is discussed for $\mathrm{BPh}-\mathrm{m}-\mathrm{APB}$ phthalonitrile system, the same approach can be used for other crosslinked networks. Simulations were carried out to investigate the coefficient of thermal expansion of the phthalonitrile model and both structural as well as electrical properties were investigated as a function of crosslink \%. Both were found to agree well with typical polymeric behavior and experimental results, a noteworthy achievement showing the potential of using MD tools for molecular simulation of polymers. These cross-linking algorithms are currently being used for the study of the carbonization process of phthalonitrile polymeric systems. Studies are also undertaken to optimize for the smallest simulation box size that is still representative of the properties of the PN polymeric system in order to investigate more material properties while saving on computational resources.

Supplementary Materials: The following are available online at www.mdpi.com/2073-4360/10/1/64/s1.

Acknowledgments: The authors would like to acknowledge and thank University of California Berkeley, Civil and Environmental Engineering department and DSO National Laboratories for funding this work and also for countless helpful discussions and support regarding the cross-linking procedure and molecular dynamics simulations.

Author Contributions: Qingsong Tu conceived of the presented idea. Janel Chua developed the cross-linking model and performed the MD simulations. Both authors contributed to the data analysis and final version of the manuscript. Qingsong Tu supervised the project.

Conflicts of Interest: The authors declare no conflict of interest.

\section{References}

1. Keller, T.M. Imide-Containing Phthalonitrile Resin. Polymer 1993, 34, 952-955. [CrossRef]

2. Zhang, L.; Liu, M.; Roy, S.; Chu, E.K.; See, K.Y.; Hu, X. Phthalonitrile-Based Carbon Foam with High Specific Mechanical Strength and Superior Electromagnetic Interference Shielding Performance. ACS Appl. Mater. Interfaces 2016, 8, 7422-7430. [CrossRef] [PubMed]

3. James, O.; Ndoke, P.N.; Kolo, S.S. Effect of Different Curing Methods on the Compressive Strength of Concrete. Available online: http:/ / www.engineeringcivil.com/effect-of-different-curing-methods-on-thecompressive-strength-of-concrete.html (accessed on 10 March 2016).

4. Kaliavaradhan, K.; Muthusamy, S. Synthesis and characterization of various phe-nylene diamine-based bismaleimide-containing phthalonitrile resins. Polym. Bull. 2016, 73, 1921-1938. [CrossRef] 
5. Yarovsky, I.; Evans, E. Computer simulation of structure and properties of cross-linked polymers: Application to epoxy resins. Polymer 2002, 43, 963-969. [CrossRef]

6. Stevens, M.J. Manipulating connectivity to control fracture in network polymer adhe-sives. Macromolecules 2001, 34, 1411-1415. [CrossRef]

7. Tsige, M.; Stevens, M.J. Effect of cross-linker functionality on the adhesion of highly cross-linked polymer networks: A molecular dynamics study of epoxies. Macromolecules 2004, 37, 630-637. [CrossRef]

8. Doherty, D.C.; Holmes, B.N.; Leung, P.; Ross, R.B. Polymerization molecular dynamics simulations. I. Cross-linked atomistic models for poly(methacrylate) networks. Comput. Theor. Polym. Sci. 1998, 8, 169-178. [CrossRef]

9. Wu, C.; Xu, W. Atomistic molecular modelling of crosslinked epoxy resin. Polymer 2006, 47, 6004-6009. [CrossRef]

10. Bandyopadhyay, A.; Valavala, P.K.; Clancy, T.C.; Wise, K.E.; Odegard, G.M. Molecular modeling of crosslinked epoxy polymers: The effect of crosslink density on thermo-mechanical properties. Polymer 2011, 52, 2445-2452. [CrossRef]

11. Heine, D.R.; Grest, G.S.; Lorenz, C.D.; Tsige, M.; Stevens, M.J. Atomistic sim-ulations of end-linked poly (dimethylsiloxane) networks: Structure and relaxation. Macromolecules 2004, 37, 3857-3864. [CrossRef]

12. Varshney, V.; Patnaik, S.S.; Roy, A.K.; Farmer, B.L. A molecular dynamics study of epoxy-based networks: Cross-linking procedure and prediction of molecular and material properties. Macromolecules 2008, 41, 6837-6842. [CrossRef]

13. Sastri, S.B.; Keller, T.M. Phthalonitrile polymers: Cure behavior and properties. J. Polym. Sci. Part A Polym. Chem. 1999, 37, 2105-2111. [CrossRef]

14. Plimpton, S.J. Fast Parallel Algorithms for Short-Range Molecular Dynamics. J. Comput. Phys. 1995, 117, 1-19. [CrossRef]

15. Materials Studio; Accelrys Software Inc.: San Diego, CA, USA, 2016.

16. Dauber-Osguthorpe, P.; Roberts, V.A.; Osguthorpe, D.J.; Wolff, J.; Genest, M.; Hagler, A.T. Structure and energetics of ligand binding to proteins: Escherichia coli dihydrofolate reductase-trimethoprim, a drug-receptor system. Proteins Struct. Funct. Genet. 1988, 4, 31-47. [CrossRef] [PubMed]

17. Burchill, P.J. On the formation and properties of a high-temperature resin from a bisphthalonitrile. J. Polym. Sci. Part A Polym. Chem. 1994, 32, 1-8. [CrossRef]

18. Keller, T.M. Phthalonitrile-based conductive polymer. J. Polym. Sci. Part A Polym. Chem. 1987, 25, $2569-2576$. [CrossRef]

19. Itecma. High Temperature Resin PN-3M. Available online: itecma.ru/en/products/svyazuyushchie/ operating-temperature-up-to-450-s/146/?type=5\#downloads (accessed on 7 May 2016).

20. Mulliken, A.D.; Boyce, M.C. Mechanics of the rate-dependent elastic-plastic deformation of glassy polymers from low to high strain rates. Int. J. Solids Struct. 2006, 43, 1331-1356. [CrossRef]

21. Richeton, J.; Ahzi, S.; Vecchio, K.S.; Jiang, F.C.; Adharapurapu, R.R. Influence of temperature and strain rate on the mechanical behavior of three amorphous polymers: Charac-terization and modeling of the compressive yield stress. Int. J. Solids Struct. 2006, 43, 2318-2335. [CrossRef]

22. Willgert, M.; Kjell, M.H.; Jacques, E.; Behm, M.; Lindbergh, G.; Johansson, M. Photoinduced free radical polymerization of thermoset lithium battery electrolytes. Eur. Polym. J. 2011, 47, 2372-2378. [CrossRef]

23. Zhao, J.; Yu, P.; Dong, S. The Influence of Crosslink Density on the Failure Be-havior in Amorphous Polymers by Molecular Dynamics Simulations. Materials 2016, 9, 234. [CrossRef] [PubMed]

24. Zhang, J. Small-scale effect on the piezoelectric potential of gallium nitride nanowires. Appl. Phys. Lett. 2014, 104, 253110. [CrossRef]

25. Ma, J.Z.; Cheng, K.; Lv, J.B.; Chen, C.; Hu, J.H.; Zeng, K.; Yang, G. Phthalonitrile-PPO Blends: Cure Behavior and Properties. Chin. J. Polym. Sci. 2017. [CrossRef]

26. Hatton, B.D.; Landskron, K.; Hunks, W.J.; Bennett, M.R.; Shukaris, D.; Perovic, D.D.; Ozin, G.A. Materials chemistry for low-k materials. Mater. Today 2006, 9, 22-31. [CrossRef]

(C) 2018 by the authors. Licensee MDPI, Basel, Switzerland. This article is an open access article distributed under the terms and conditions of the Creative Commons Attribution (CC BY) license (http:// creativecommons.org/licenses/by/4.0/). 